\title{
STABILIZATION OF KINK INSTABILITIES BY EDDY CURRENTS IN A SEGMENTED WALL AND COMPARISON WITH IDEAL MHD THEORY
}

\author{
A.M. GAROFALO, E. EISNER, T.H. IVERS, R. KOMBARGI, M.E. MAUEL, D. MAURER, \\ D. NADLE, G.A. NAVRATIL, M.K. VIJAYA SANKAR, E. TAYLOR, Q. XIAO \\ Department of Applied Physics, \\ Columbia University, \\ New York, N.Y., \\ United States of America
}

ABSTRACT. The characteristics of external kink instabilities observed during wall stabilization studies in the HBT-EP tokamak have been compared with the predictions of ideal MHD theory, in order to examine the stabilizing role of a resistive wall that is segmented both toroidally and poloidally. The reconstructed equilibria, for discharges with different plasma-wall configurations, are consistent with external and internal magnetic measurements, measured soft X ray profiles and measured equilibrium wall eddy currents. The stability analysis of these equilibria predicts patterns of instability induced eddy currents for a model wall that is continuous and perfectly conducting, and these patterns are in good agreement with the ones observed on the HBT-EP segmented wall. These eddy currents account for the observed stabilization of fast ideal modes when the wall is fully inserted, consistent with the prediction of marginal stability.

\section{INTRODUCTION}

In most tokamaks, the external ballooning kink instability sets the most severe limit on beta (the ratio of plasma pressure to magnetic field pressure). This limit is rather well reproduced by the following simple scaling law:

$\beta_{\mathrm{N}}=\frac{\beta(\%)}{I_{\mathrm{p}} / a B_{\phi}} \leq C$

where $\beta_{\mathrm{N}}$ is the normalized beta, $I_{\mathrm{p}}$ is the plasma current, $a$ is the minor radius, $B_{\phi}$ is the toroidal magnetic field and the value of $C$ is $2.8 \mathrm{MA} /(\mathrm{m} \cdot \mathrm{T})$ as originally identified by Troyon [1] from numerical and experimental evidence.

Since an external mode requires motion of the plasma-vacuum interface away from its equilibrium position, the ideal recourse to overcome such instability would be to place a perfectly conducting wall surrounding the plasma and close to its surface [2]. In this way the eddy currents generated in the wall by the plasma displacement can react back on the mode and neutralize it.

Wall stabilization is particularly important for the so-called 'advanced tokamak regimes', which refer to plasma equilibria having high beta, high energy confinement time and high 'bootstrap' current (i.e. the non-inductive, self-generated toroidal plasma current, favourable for steady state operation). This is the case of the advanced regimes calculated for TPX [3], NSTX [4], ARIES-II and ARIES-IV [5], and DIII-D [6], which rely on a close fitting perfectly conducting wall to stabilize the $n=1$ external ballooning kink mode.

In addition, wall stabilization may be important for more conventional tokamak discharges. Sensitivity studies on safety factor profiles for conventional operating modes in ITER have found coupled internalexternal kink modes unstable at very low beta values [7]. These modes can be stabilized by a perfectly conducting wall.

Owing to finite dissipation in any real, nonsuperconducting wall, the eddy currents can only slow down external instabilities in a non-rotating plasma to the order of a characteristic penetration time of the wall. In this case the modes are usually called 'wall modes', and their slow growth rates may allow feedback techniques to provide stabilization in steady state $[8-11]$.

In contrast, when the plasma rotates sufficiently, theoretical work [12-15] and computational stability models [16] that included dissipation in the plasma suggest that a resistive wall can provide long term stability without active feedback control. Experimental results in DIII-D [17] and PBX-M [18] have found a possible explanation in these resistive wall mode theories. 


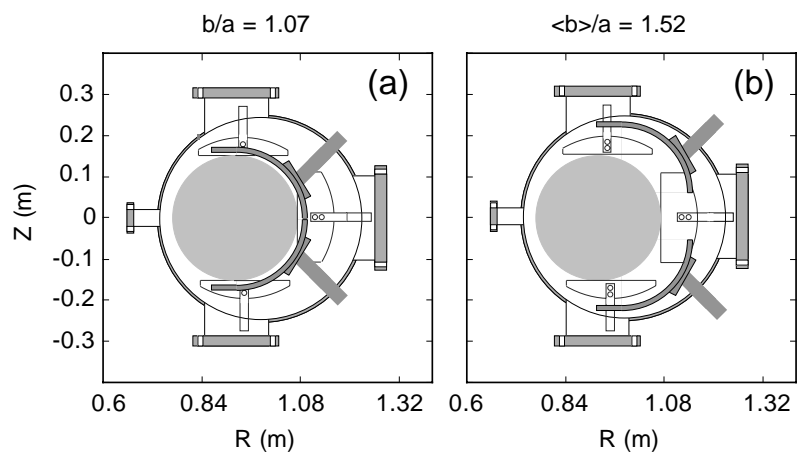

FIG. 1. Schematic cross-section of HBT-EP, showing poloidal limiters and conducting wall in the (a) fully inserted and (b) fully retracted positions.

The HBT-EP experiment at Columbia University [19] was designed and built to make possible a systematic study of the effects of a resistive wall on the stability of the kink mode. The internal conducting wall in HBT-EP is segmented so that it can be moved independently from the surface of the plasma, permitting variations of the plasma-wall distance and of the symmetry and fraction of the toroidal coverage, while the other plasma parameters are kept nearly identical. In this article we focus on the examination of the eddy current patterns induced on the internal wall by MHD instabilities during wall stabilization experiments. We have carefully reconstructed the HBT-EP equilibria to compare the experimental observations with the predictions of ideal MHD theory. The simulations show that achievable beta values reach beyond the stability limit to external ballooning kink modes calculated in the absence of the wall ('no-wall limit'). The similarities and differences between the instability induced eddy current patterns on the actual wall and on the model wall provide important information on the wall stabilization mechanism when a segmented wall is used. This study allows us to conclude that the segmentation of the wall does not prevent the eddy currents from reproducing the helical patterns predicted for a continuous wall and that these eddy currents can account for the stabilizing effects observed when the wall is fully inserted.

The outline of the article is as follows: in Section 2 we describe briefly the HBT-EP tokamak and in Section 3 the numerical tools used for the simulations. In Section 4 we present the experimental results and compare them with the numerical predictions. In Section 5 we discuss the conclusions.

\section{THE HBT-EP EXPERIMENT}

The vacuum chamber of HBT-EP is composed of ten stainless steel sections, each of which supports a pair of conducting wall segments. Some of the major characteristics and typical parameters of the HBT-EP tokamak are given in Table I; more detailed descriptions of the HBT-EP device are given in Refs $[19,20]$. The wall segments are made of $1.2 \mathrm{~cm}$ thick aluminium (nickel plated to minimize sputtering), and the decay time of the wall eddy currents, measured during penetration of the vacuum vertical field, is $\tau_{L / R} \approx 7.8 \pm 1.2 \mathrm{~ms}$. Each segment can be independently moved in a range of $8 \mathrm{~cm}$ along a radial direction at $45^{\circ}$ with respect to the outer mid-plane of the torus, as shown in Fig. 1. When fully inserted (Fig. 1(a)) the wall conforms closely to the plasma outer side. In this configuration the distance of the wall from the plasma surface is about $1 \mathrm{~cm}(b / a=1.07)$ and the toroidal angle covered by each segment is $28^{\circ}$. The toroidal gaps between wall segments can be thought of as comparable to the openings that in large devices are needed for the access to the plasma by diagnostics and particle beams. When the wall is fully retracted (Fig. 1(b)) the plasma minor radius can be varied by retracting the stainless steel poloidal limiters. Numerical calculations show that for ideal MHD stability the plasmawall configuration of Fig. 1(b) $(\langle b\rangle / a=1.52)$ is nearly equivalent to having the wall infinitely far from the plasma [20].

Table I. Major Characteristics and Typical Parameters of HBT-EP

( $R$ is the major radius, $b$ is the radial distance of the wall from the plasma centre, $\epsilon^{-1}$ is the aspect ratio, $q$ is the safety factor, $n_{\mathrm{e}}$ is the electron density and $T_{\mathrm{e}}$ is the electron temperature)

\begin{tabular}{|c|c|c|}
\hline \multirow[b]{2}{*}{ Parameter } & \multicolumn{2}{|c|}{ HBT-EP } \\
\hline & Wall fully inserted & Wall fully retracted \\
\hline$R(\mathrm{~m})$ & 0.92 & $0.92-0.94$ \\
\hline$a(\mathrm{~m})$ & 0.15 & $0.15-0.20$ \\
\hline$b / a$ & 1.07 & $1.52-1.05$ \\
\hline$\epsilon^{-1}$ & 6.13 & $6.13-4.7$ \\
\hline$\beta_{\mathrm{N}}$ & \multicolumn{2}{|c|}{1.5} \\
\hline$q_{\text {edge }}$ & \multicolumn{2}{|c|}{$2-4$} \\
\hline$I_{\mathrm{p}}(\mathrm{kA})$ & \multicolumn{2}{|c|}{16} \\
\hline$B_{\phi}(\mathrm{kG})$ & \multicolumn{2}{|c|}{3.5} \\
\hline$n_{\mathrm{e}}\left(\mathrm{m}^{-3}\right)$ & \multicolumn{2}{|c|}{$1 \times 10^{19}$} \\
\hline$T_{\mathrm{e}}(\mathrm{eV})$ & \multicolumn{2}{|c|}{100} \\
\hline
\end{tabular}



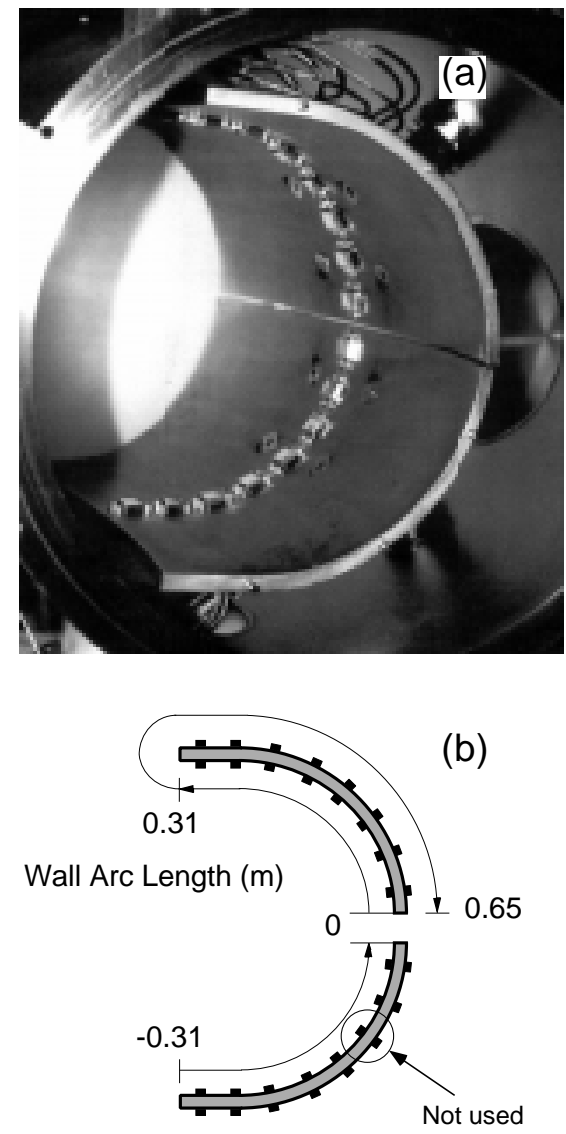

FIG. 2. (a) Photograph and (b) schematic cross-section of the wall segments carrying the wall mounted probe array. The solid boxes represent the stainless steel shields enclosing the Mirnov coils. The coils at the locations circled were shorted to the wall and not used. The arc length convention shown here is used throughout this article.

At two opposite toroidal locations the wall segments are instrumented with arrays of magnetic coils that measure the magnetic field at the wall in the poloidal and radial directions. Figure 2 shows the configuration of the wall mounted probe array used for the measurements reported in this article. Using a local slab approximation, the toroidal component of the current per unit length at the locations between a coil on the plasma facing side (in) and a coil on the vacuum side (out), can be calculated as

$j_{\phi}=\left(B_{\theta, \text { out }}-B_{\theta, \text { in }}\right) / \mu_{0}$

where the positive sign for $j$ is aligned with the plasma current. Because of toroidal rotation of the plasma, magnetic signals from MHD activity in HBTEP show fluctuations that usually have frequencies greater than $4 \mathrm{kHz}$. If we then decompose the magnetic signal, $B$, in a 'perturbed' part, $\delta B$, and an 'equilibrium' part, $\langle B\rangle$, we assume that the perturbed part does not penetrate the wall because at frequencies $\geq 4 \mathrm{kHz}$ the skin depth of aluminium is at most $1 \mathrm{~mm}$, much smaller than the thickness of the wall of HBT-EP. Therefore from the measurement of $\delta B$ on each side of the wall we obtain the instability induced eddy current on each side of the wall

$$
\delta j_{\phi, \text { in }}=-\delta B_{\theta, \text { in }} / \mu_{0}, \quad \delta j_{\phi, \text { out }}=\delta B_{\theta, \text { out }} / \mu_{0} .
$$

\section{NUMERICAL MODELLING}

\subsection{Equilibrium}

The numerical code used for the reconstruction of the equilibria presented in this article implements a standard technique [21] that computes the coefficient in polynomial expansions of the equilibrium profiles by a least squares fit to experimental measurements. An equilibrium reconstruction with representative profiles is shown in Fig. 3.

Our set of measurements includes data from an internal magnetic probe that reaches three centimetres into the plasma edge, the wall mounted probes that measure the tangential magnetic field at the conducting wall, an array of flux loops and Rogowski coils that measure the equilibrium coil currents and the plasma current and position (Fig. 3(a)). To this information we add the assumption that the safety factor at the magnetic axis, $q_{0}$, is $\approx 1$ during sawtoothing periods of plasma discharges. This is a conventional approach that has been used in several previous stability studies (e.g., Ref. [22]). In support of this procedure, it has been shown that experimentally observed stability boundaries for sawtoothing discharges agree well with the results of stability calculations performed on equilibrium reconstructions having $q_{0}$ artificially raised to just above 1 [23, 24].

A challenge arises in HBT-EP from the necessity of including in the reconstructions the presence of axisymmetric $(n=0)$ eddy currents in the internal wall segments, since the decay time of these eddy currents is comparable to the discharge duration. Also important are the axisymmetric eddy currents induced in the vacuum chamber segments and the two inches thick aluminium cylinder, with two toroidal breaks used to oppose the centring forces of the toroidal field magnets.

We represented the equilibrium eddy currents flowing in the conducting structures with sets of toroidally symmetric coils, or filaments, having zero net current, as illustrated in Fig. 3(a). For the adjustable wall and the vacuum chamber, we 

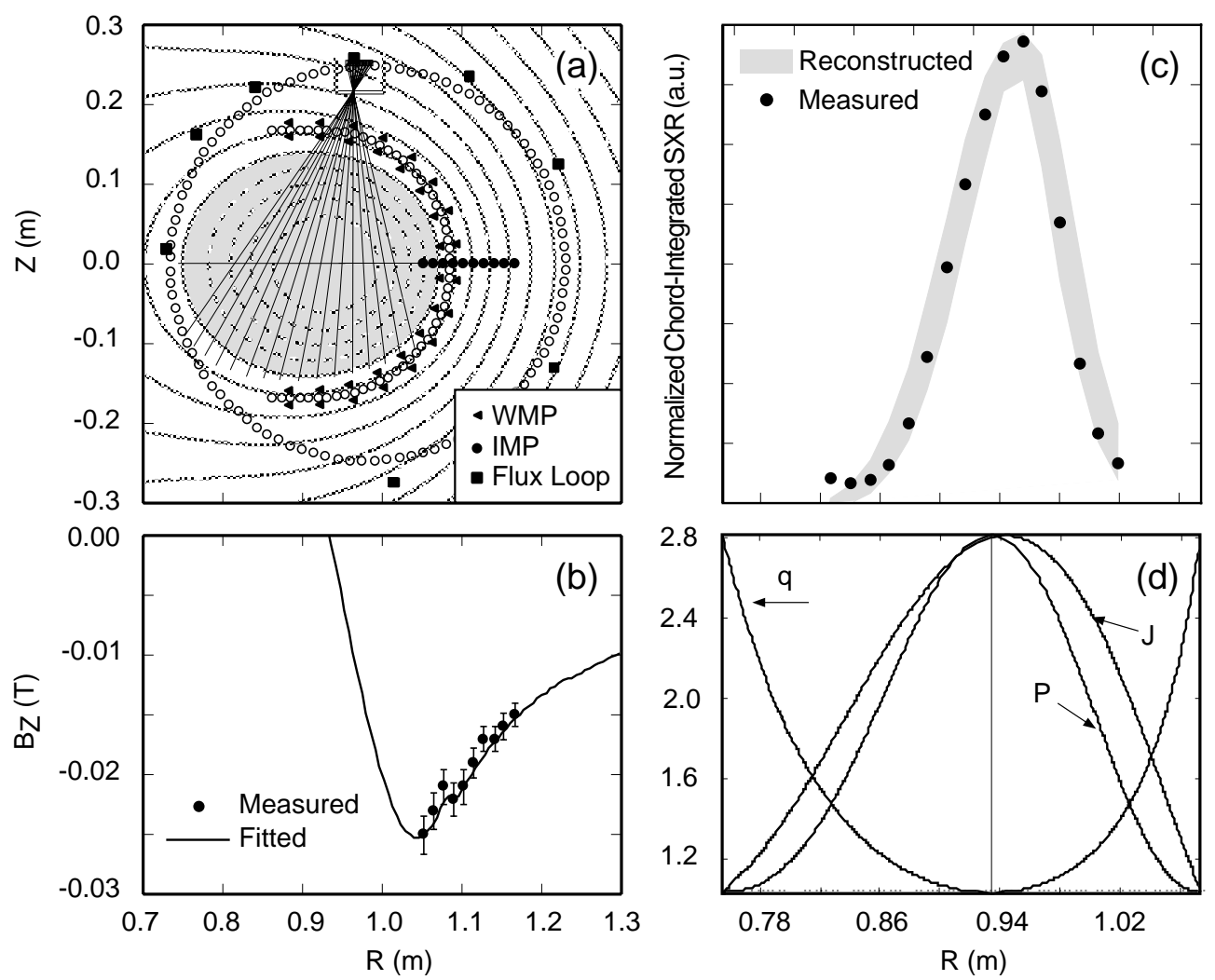

FIG. 3. Measured profiles and equilibrium reconstruction for discharge 11491 at $t=3.2 \mathrm{~ms}$ : (a) poloidal flux surfaces with magnetic measurement locations (WMP, wall mounted probe; IMP, internal magnetic probe), soft X ray viewing chords and coil representation of conducting structures; (b) measured and fitted profiles of vertical field at IMP locations; (c) measured and simulated profiles of chord integrated SXR emission; (d) safety factor, current density and pressure from equilibrium reconstruction.

decomposed the poloidal distribution of the toroidal eddy currents in orthonormal eigenmodes, as was done for the vessels in the ISX-B and DIII-D tokamaks [25]. The currents that flow in these eigenmodes are computed self-consistently with the equilibrium currents that flow in the plasma.

The eigenvalues of the decomposition equal the $R / L$ decay rates for the corresponding circuits. Each subsequent mode has a shorter spatial scale length than the next lower order mode and, therefore, a faster decay. Figure 4 illustrates the current distribution for the first three up-down symmetric eigenmodes with no net toroidal current calculated for the upper segment of the adjustable wall. The arc length is measured with the origin on the outboard midplane.

The calculated decay times can be corrected to include the effect of multiple toroidal breaks using the model described by Nagayama et al. for the tokamak TNT-A [26]. Table II summarizes the decay times of

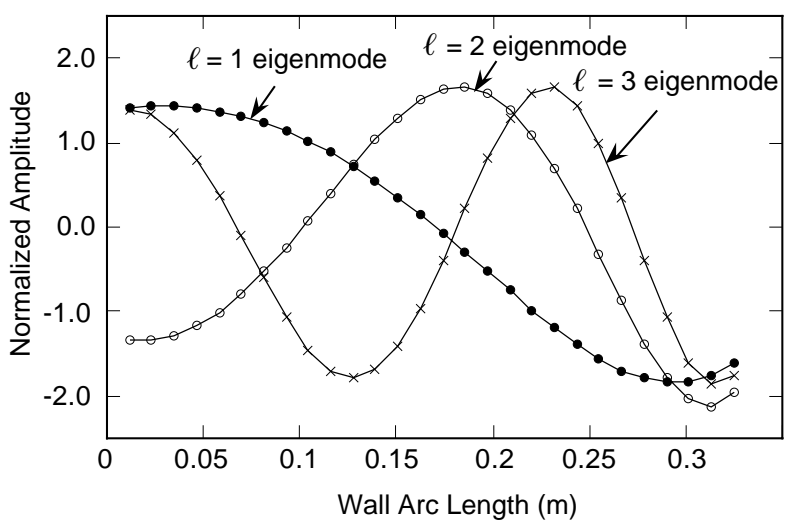

FIG. 4. First three up-down symmetric eigenmodes of the poloidal distribution of the wall eddy currents.

the $l=1,2,3$ eigenmodes for the fully inserted wall and the $l=1$ eigenmode for the vacuum chamber. The decay time of $12 \mathrm{~ms}$ calculated for the $l=1$ wall eigenmode is in reasonable agreement with the 
Table II. Calculated Decay Times for $n=0$ Eddy Current Eigenmodes in the Conducting Wall and the Vacuum Chamber

\begin{tabular}{lccc}
\hline $\begin{array}{c}\text { Eigenmode order } \\
\text { (up-down symmetry) }\end{array}$ & $\begin{array}{c}L / R(\mathrm{~ms}) \\
\text { One toroidal break }\end{array}$ & $\begin{array}{c}L / R(\mathrm{~ms}) \\
\text { Ten toroidal breaks }\end{array}$ \\
\hline Conducting wall & $l=1$ & 23 & 12.2 \\
& $l=2$ & 13 & 6.9 \\
Vacuum chamber & $l=3$ & 8.4 & 4.5 \\
\hline
\end{tabular}
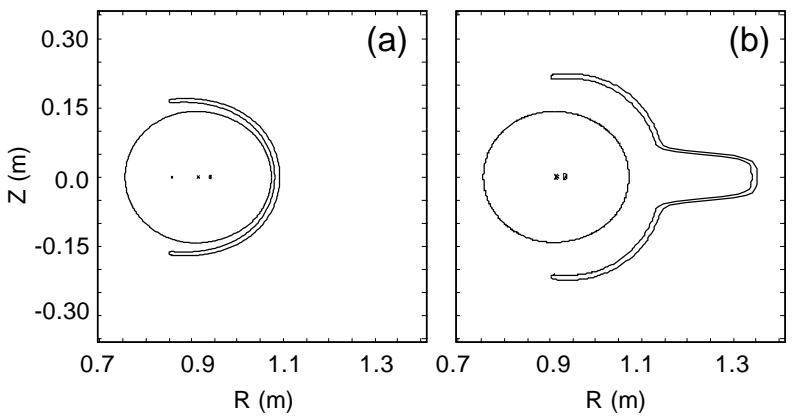

FIG. 5. VACUUM model for the wall of HBT-EP in (a) the wall fully inserted configuration and (b) the wall fully retracted configuration.

measured decay time for vertical field penetration. The decay times for the corresponding $n=1$ distributions can be expected to be somewhat shorter.

The total equilibrium toroidal eddy current pattern in a set of coils can be prescribed in the equilibrium code as a linear combination of several eigenmodes. The magnitudes of each eigenmode are computed as those best fitting the totality of the measurements.

Using the reconstructed equilibrium, we have simulated the signals measured by our soft X ray detector array, assuming a uniform plasma density. A comparison between the measured and simulated bremsstrahlung profile is illustrated in Fig. 3(c). The comparison shows good agreement, providing evidence supporting the accuracy of both the reconstructed current profile and the reconstructed pressure profile.

\subsection{Stability}

The ideal MHD stability of the reconstructed equilibria has been tested with the PEST routines [27, 28], interfaced with the VACUUM [29] code to incorporate the solution to Laplace's equation in the vacuum. Presently, VACUUM is the only ideal MHD code able to model the case of a plasma with a surrounding wall having poloidal structure. The use of VACUUM allows one to simulate the HBT-EP wall with a toroidally symmetric, perfectly conducting wall, with one poloidal gap and a deformable bulge on the outer major radius. Figure 5 shows the wall model used to approximate the two experimental configurations: (a) wall fully inserted and (b) wall fully retracted.

If an instability is found, the eddy current pattern induced on the wall can be calculated and displayed. Figure 6(a) shows the predicted pattern of the toroidal component of the eddy currents induced by an $n=1, m=2$ external kink mode in the configuration with the wall fully inserted (the model wall shown in Fig. 6(b)). On the plasma facing side of the wall the pattern mirrors the $n=1, m=2$ helix. The eddy currents calculated on the vacuum side of the wall are much less coupled to the plasma and are therefore 1 order of magnitude smaller than the currents on the plasma facing side of the wall. The calculations show that the paths of lowest inductance for these small toroidal currents on the vacuum side of the wall run almost purely along the poloidal direction.

\section{EXPERIMENTAL RESULTS AND COMPARISONS WITH IDEAL MHD}

The plasma discharges in the experiments described in this article have been designed to reach $\beta_{\mathrm{N}}$ near or above the critical $\beta_{\mathrm{N}}$ for stability to the ideal MHD external kink mode predicted without a wall. With an edge safety factor, $q_{\text {edge }}$, between 2 and 3 , calculations conducted using standard monotonic HBT-EP plasma profiles [20] showed that the predicted no-wall $\beta_{\mathrm{N}}$ limit to an $n=1$ external kink mode drops to values near 1.5 , i.e. values achievable in HBT-EP with ohmic heating only. To further lower the no-wall $\beta_{\mathrm{N}}$ limit, we planned to broaden the 


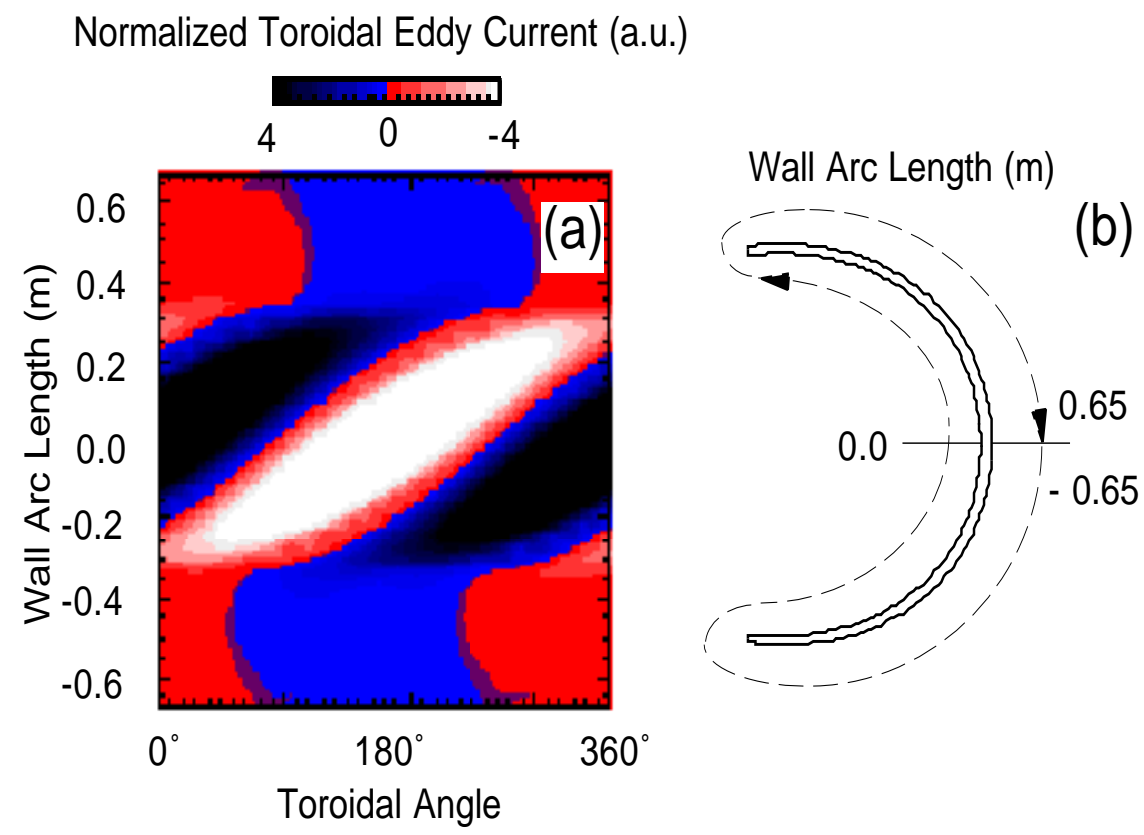

FIG. 6. (a) Toroidal eddy current pattern calculated by PEST-VACUUM for the case of an $m=2, n=1$ external kink mode with the 'wall fully inserted' model wall. (b) The wall arc length is zero on the plasma facing side of the wall, at the midplane; positive in the direction of the arrow up to the midplane on the vacuum side of the wall.

plasma current profile by maintaining a positive current ramp throughout most of the plasma discharges. These relatively high beta and broad current profile plasmas are created by programming a short period of rapid current startup $\left(\partial I_{\mathrm{p}} / \partial t \sim 100 \mathrm{MA} / \mathrm{s}\right)$ followed by a moderate current ramp $\left(\partial I_{\mathrm{p}} / \partial t \sim 3 \mathrm{MA} / \mathrm{s}\right)$. In order to maintain as closely as possible a constant edge safety factor, the vertical field increases with the plasma current, causing the magnetic axis to move away from the outer radial limiter and the minor radius to increase. In Fig. 7 the plasma current and safety factor time evolutions are compared for discharges with the wall fully inserted and with the wall fully retracted. The shaded areas denote the time periods of interest for the stability study.

Because the discharges exhibit sawtoothing oscillations the assumption of $q_{0} \approx 1$ is used in the reconstruction of the equilibria. This equilibrium reconstruction procedure has been applied to same minor radius plasmas formed with the wall fully retracted and with the wall fully inserted. The reconstructions are performed for time points at which discharges with different plasma-wall configuration reach similar values in $\beta_{\mathrm{N}}$ and $q_{\text {edge }}$, but whose unstable behaviour evolves in different ways.
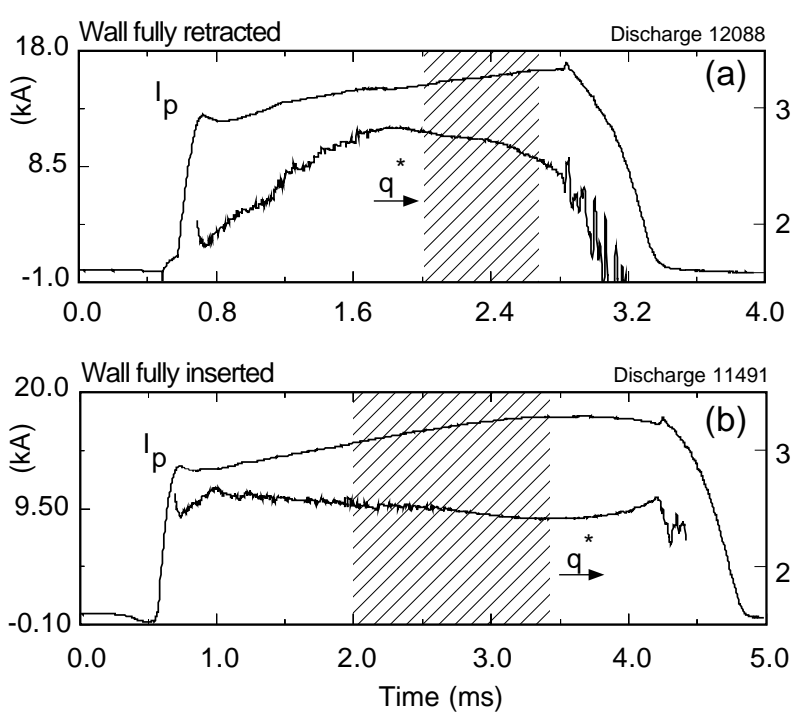

FIG. 7. Time evolution of plasma current and cylindrical safety factor for discharges formed with (a) the wall fully retracted and (b) the wall fully inserted.

\subsection{Stability boundary}

In discharges with the wall fully retracted, a rapidly growing precursor (growth time $\gamma^{-1} \leq$ $200 \mu \mathrm{s})$, which appears rotating with frequency 


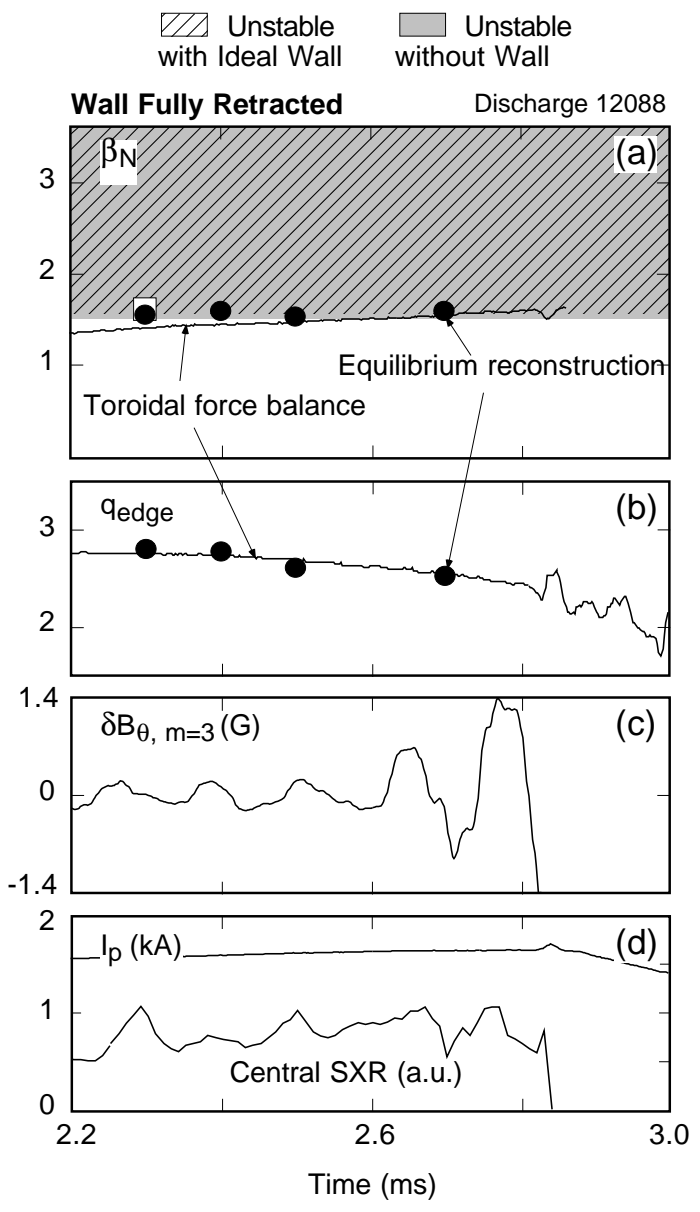

FIG. 8. Disruptions induced by MHD instabilities in discharges with the wall fully retracted: (a) normalized beta, (b) edge safety factor, (c) $m / n=3 / 1$ magnetic fluctuations, (d) plasma current and central chord of the soft $\mathrm{X}$ ray diagnostic. Discharge 12088 . The solid circles represent values from equilibrium reconstructions. The hollow symbol represents the companion flux conserved equilibrium with increased beta, unstable with an ideal wall. The shaded areas indicate the predicted stability boundaries calculated with and without an ideal wall at $t=2.3 \mathrm{~ms}$.

$f_{\text {mode }} \approx 8 \mathrm{kHz}\left(\sim 7 \%\right.$ of the Alfvén frequency, $\omega_{\mathrm{A}}=$ $\left.V_{\mathrm{A}} / q R\right)$, results in a current disruption when $q_{\text {edge }}$ decreases below 3 and $\beta_{\mathrm{N}}$ reaches values close to 1.5 (Fig. 8). The values of $\beta_{\mathrm{N}}$ and $q_{\text {edge }}$ from equilibrium reconstructions are indicated in Figs $8(\mathrm{a})$ and (b) by solid circles. These values are in good agreement with the time evolutions of the same quantities estimated from toroidal force balance using magnetic measurements of the poloidal beta [30], and shown in the same figures as the solid traces. The critical $\beta_{\mathrm{N}}$ for stability to the $n=1$ external kink predicted by the PEST-VACUUM codes in the presence of the ideal wall (the cross-hatched area in Fig. 8(a)) and with-

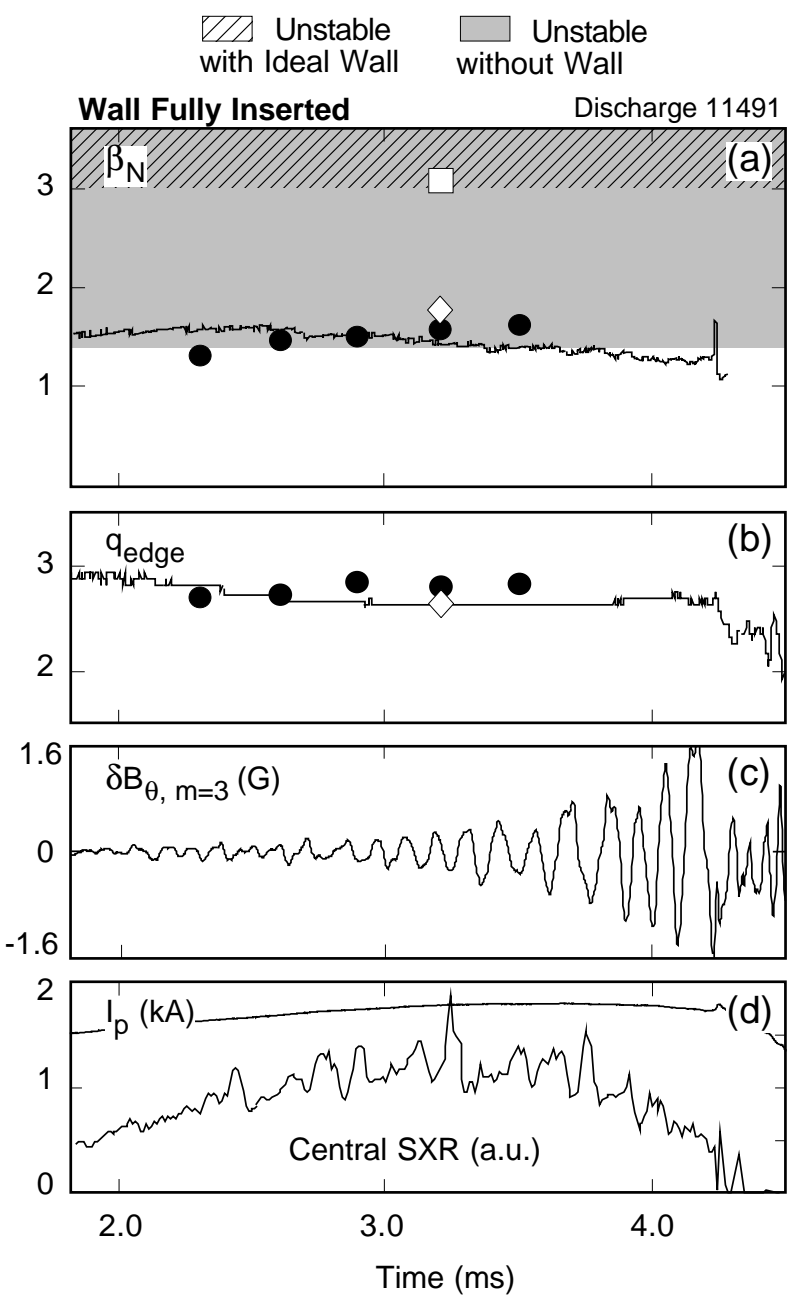

FIG. 9. Slowly growing precursors in discharges with the wall fully inserted. Hollow symbols represent companion equilibria unstable with the ideal wall, generated by increasing $\beta_{\mathrm{N}}$ (squares) and by decreasing the magnetic shear (diamonds). Predicted stability boundaries are calculated at $t=3.2 \mathrm{~ms}$. Discharge 11491.

out the ideal wall (the gray shaded area in Fig. 8(a)) are almost coincident. This shows that completely retracting the wall produces a good approximation to a configuration with no conducting wall in HBT-EP. In good agreement with the experiment, the no-wall ideal MHD calculations predict an unstable global external mode with growth time $\gamma_{\text {PEST }}^{-1} \approx 90 \mu$ s at the time when the growth of the precursor starts to occur.

In discharges with the wall fully inserted (Fig. 9), the $\beta_{\mathrm{N}}$ stability limit to $n=1$ external kink is predicted to double in the presence of the ideal wall. As $\beta_{\mathrm{N}}$ approaches 1.5 with $q_{\text {edge }}$ just below 3 , the discharges exceed the marginal stability boundary calculated without the ideal wall. However, the fast 


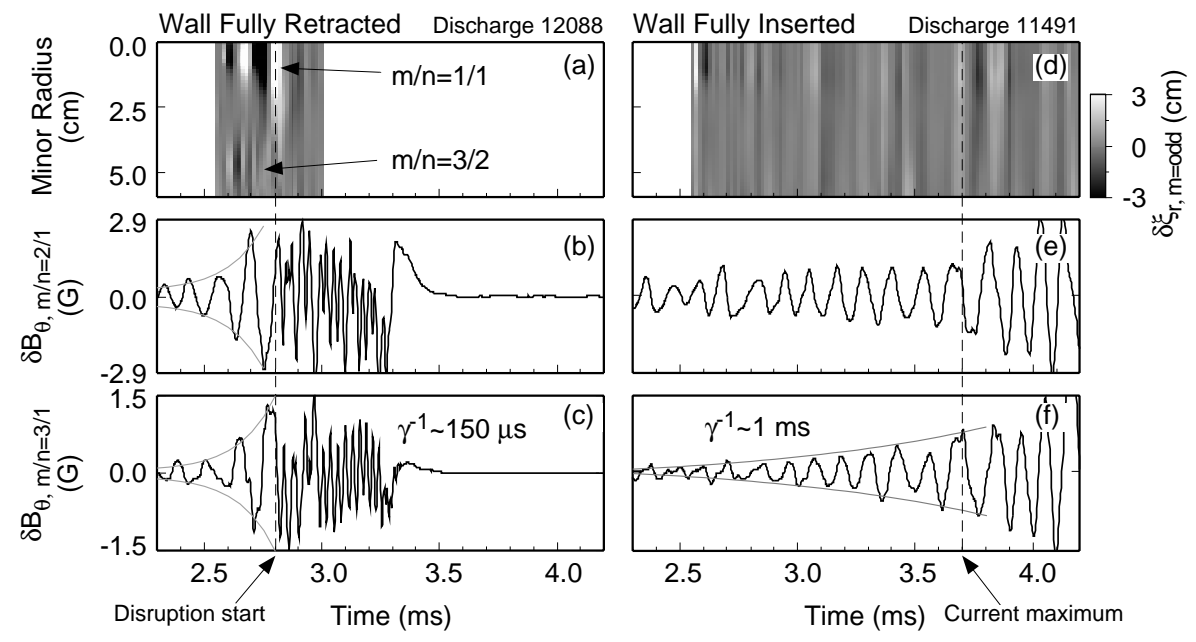

FIG. 10. Comparison of mode structure measurements for discharges with the wall fully retracted (12088) and fully inserted (11491). (a, d) Radial plasma displacement for odd- $m$ components in the core region; (b, e) $m=2$ component and (c, f) $m=3$ component of the perturbed poloidal magnetic field measured by Fourier analysing Rogowski coils.

growing ideal instability predicted without the wall $\left(\gamma_{\mathrm{PEST}}^{-1} \approx 30 \mu \mathrm{s}\right.$ at $\left.t=3.2 \mathrm{~ms}\right)$ appears to be stabilized in the experiment, in good agreement with the elevated $\beta_{\mathrm{N}}$ stability limit predicted for the ideal kink mode in the presence of the ideal wall.

Figure 9 shows that, when the wall is fully inserted, slowly growing modes are destabilized when $\beta_{\mathrm{N}}$ crosses the stability boundary predicted in the absence of the wall. The growth time of these modes is close to the wall penetration time during the postive $\partial I_{\mathrm{p}} / \partial t$ period $\left(\gamma^{-1} \sim 1 \mathrm{~ms}\right)$, and decreases to about $600 \mu$ s after the plasma current reaches its maximum. In the following section, a more detailed examination of the mode structure will show that these precursors are different in nature from the fast ideal modes observed when the wall is fully retracted.

\subsection{Mode structure}

In both cases of wall fully retracted and wall fully inserted discharges, measurements from toroidally distributed Fourier analysing Rogowski coils (a type of Rogowski coil but with effective winding density varying as $\cos m \theta$ or $\sin m \theta$ [31]) and Mirnov loops identify the disruption precursor as an $n=1$ mode, with a large $m=3$ component calculated to have a resonant $q$ surface in the vacuum. Figure 10 shows a comparison of our measurements of the various poloidal components in the cases with the wall fully retracted and with the wall fully inserted. The odd- $m$ components of the plasma radial displacement in Figs 10(a) and (d) are estimated from the fluctuating part of the chord integrated soft $\mathrm{X}$ ray intensity profile $\delta S(r, t)$, similarly to Ref. [32]: $\delta S(r, t)$ is decomposed into symmetric and antisymmetric components with respect to the magnetic axis, then the latter is normalized by the gradient of $\langle S(r, t)\rangle$, the equilibrium part of the profile. Given our viewing solid angle, the displacement $\xi_{r}(r, t) \approx$ $\delta S_{\text {asym }}(r, t) / \nabla\langle S(r, t)\rangle$ spans only the plasma core. The fluctuation localized near the magnetic axis, where we assume that $q \approx 1$, is identified as a mode number $m=1$. The $m=2$ and $m=3$ fluctuations in the poloidal magnetic field, $\delta B_{\theta}$, are detected at the same minor radius by Fourier analysing Rogowski coils. Figures $10(\mathrm{a})$ to (c) show that in discharges with the wall fully retracted the disruption precursor is a global instability with $m / n=1 / 1,2 / 1$ and $3 / 1$ components. The $m=2$ and $m=3$ components are shown to grow rapidly and at the same rate, leading to a disruption during the positive $\partial I_{\mathrm{p}} / \partial t$ period. The similar fluctuation frequency for the measured $n=1$ components, together with observations of $m / n=3 / 2$ fluctuations having twice the frequency of the $n=1$ components, suggest rigid toroidal rotation of these plasmas.

In comparison, the instabilities seen in discharges with the wall fully inserted have a less global character, with a smaller or no $m=1$ component (Fig. 10(d)). Furthermore, the phase relationship 

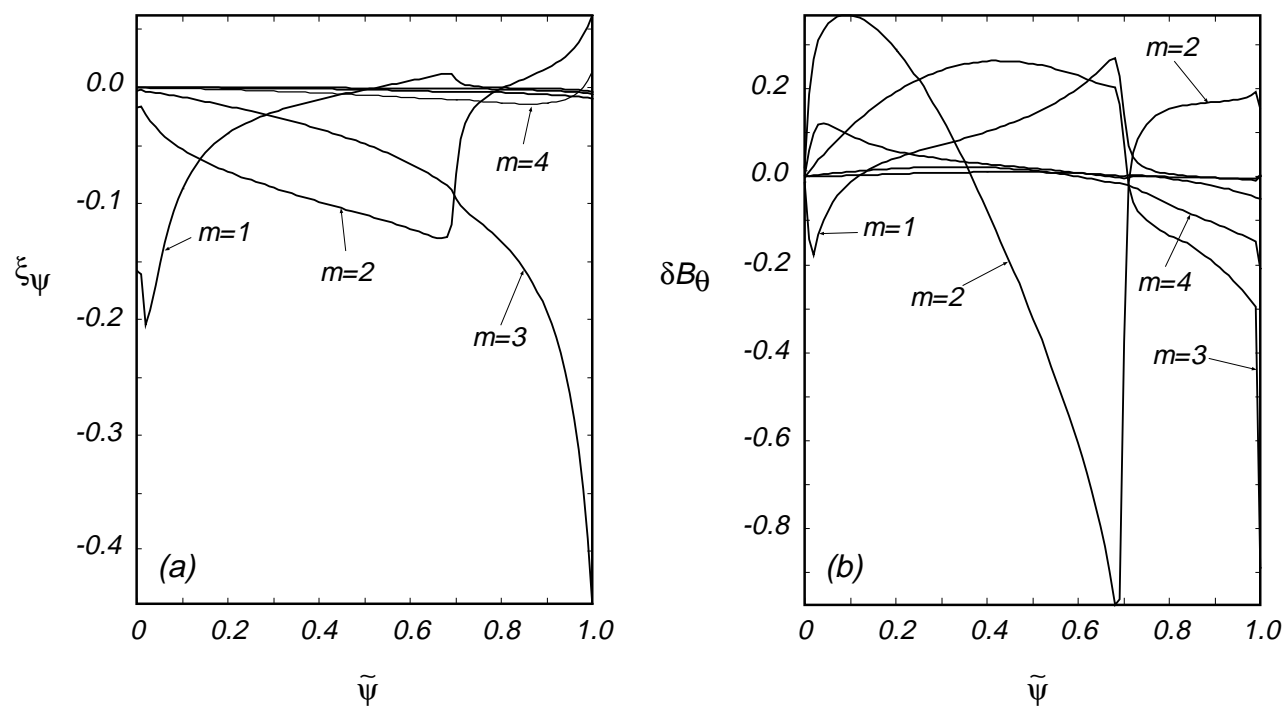

FIG. 11. Instability calculated by PEST1 for discharge 12088 without an ideal wall. Radial dependence of different Fourier components of (a) the normal plasma displacement and (b) the perturbed poloidal magnetic field.

between the $m=3$ and $m=2$ Rogowski coil signals, which is $\approx-150^{\circ}$ for the wall retracted cases, has changed to $\approx-70^{\circ}$ for the wall fully inserted cases (Figs 10(e) and (f)). Often, in these wall inserted cases, the $m=2$ and $m=3$ components appear to have different time histories: during the positive $\partial I_{\mathrm{p}} / \partial t$ period the $m=2$ component has a nearly constant amplitude, while the $m=3$ component grows on a time-scale close to the wall penetration time. After the plasma current maximum, the negative current ramp produces a more peaked current profile and might therefore destabilize tearing modes driven by internal current gradients [20]. During this period, the $m=2$ and $m=3$ components grow together at an increased rate and lead to the disruption.

Figure 11 shows the radial structure of the unstable eigenfunction calculated by PEST1 without the ideal wall, for the discharge with the wall fully retracted of Figs 10(a) to (c). This no-wall eigenfunction is nearly identical to the no-wall eigenfunction predicted for the discharge with the wall fully inserted of Figs 10(d) to (f) (the equilibria are nearly identical, the wall position is different). It can be seen that the instability predicted in the absence of a wall consists of a mix of $m$-numbers, spanning the crosssection of the plasma. From Fig. 11(b) one can calculate the ratio of the $m=3 / m=2$ components of the perturbed poloidal field at the plasma surface: $\delta B_{\theta, m=3} / \delta B_{\theta, m=2} \approx 5$. Using the behaviour $\delta B_{\theta} \propto$ $m / r^{m+1}$ for the perturbed poloidal field in the vac- uum, one obtains at the radius of the Rogowski coils $(\sim 12 \mathrm{~cm}$ from the plasma surface) a predicted ratio $\delta B_{\theta, m=3} / \delta B_{\theta, m=2} \approx 0.6$. This value is in good agreement with the ratio from the measurements made during the wall retracted case, shown in Figs 10(b, c).

\subsection{Eddy currents}

Figures 12(a) and (b) show the time evolution of the toroidal component of the instability induced eddy currents measured on the plasma facing side of the wall for a wall fully retracted discharge, around the time of the disruption. There are 15 measurements distributed on the plasma facing side of the two instrumented wall segments of Fig. 2. The images of the measued eddy current patterns in Figs 12(b) and (c) are obtained by linearly interpolating between measurements. This method allows a clear representation of the helical perturbation growing as it passes by the poloidal array of probes at nearly constant frequency until the disruption at about $t=2.8 \mathrm{~s}$.

Given the rigid toroidal rotation of the plasma, we can directly compare the eddy currents that are PEST-VACUUM calculated over one period in the toroidal angle (Fig. 12(d)) with the measurements taken over a time interval $\tau=1 / f_{\text {mode }}$ (Fig. 12(c)).

When the codes find stability in the presence of the ideal wall, to obtain the eddy current pattern induced by kink modes for the comparison we have either scaled up the pressure in flux conserved equilibria [33], or recalculated the equilibrium with increased 


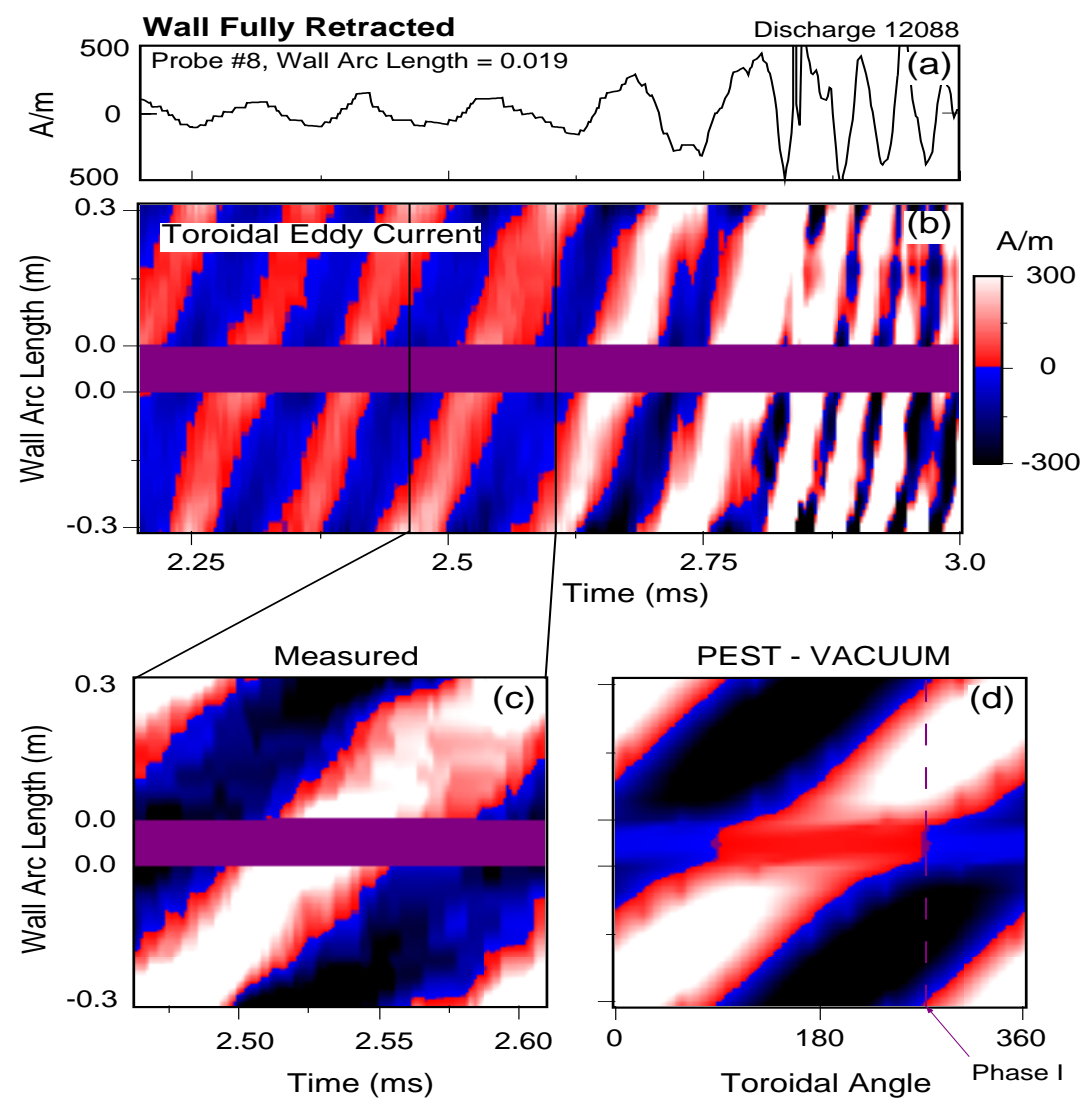

FIG. 12. Toroidal component of the instability induced eddy currents on the plasma facing side of two wall segments during discharges with the wall fully retracted: (a, b, c) time evolution; (d) pattern calulated by PEST1-VACUUM. Discharge 12088.

$q$ on-axis. These unstable 'companion' equilibria are represented by hollow symbols in Figs 8 and 9. The eddy current pattern from these companion equilibria does not vary significantly as long as $q_{\text {edge }}$ is not changed. In the wall retracted case the $3 \%$ increase in $\beta_{\mathrm{N}}$ for the companion equilibrium is within the uncertainties of the calculations for the original reconstruction. The exact matching of the pitch of the measured and calculated current patterns confirms the presence of a large $m=3$ component in the observed instabilities. As a sensitivity check, we have calculated the eddy current patterns for instabilities in companion equilibria having lower $q_{\text {edge }}$, so that the dominant poloidal component at the edge is $m=2$. These patterns show a more vertical pitch that disagrees with the measurements.

To carry out our comparisons in more detail, we have picked a phase of the poloidal distribution of the toroidal eddy current (phase I in Fig. 12(d)) and compared the code calculations with the average of measured distributions having the same phase at dif- ferent time points. The amplitudes of the measured eddy current have been scaled for comparison with the theoretical profiles.

Figure 13(a) shows good general agreement for wall retracted, with deviations towards the outboard gap (a wall arc length of 0 ), where the bulge in the model wall allows the eddy currents to smoothly go to zero at the midplane. Figure 13(b) shows the analogous comparison for the wall inserted discharge, with good general agreement except towards the edges by the inboard gap, for reasons that will be discussed later.

Figure 14 shows for a wall inserted discharge, the eddy currents measured by all 16 probes in the top wall segment (Fig. 2). The measured change in pattern from the plasma facing to the vacuum side of the wall corresponds to the predictions for the ideal wall, confirming that the instability induced eddy currents do not leak through the wall. The vacuum side of the wall is shielded from the helical instability by the plasma facing side of the wall. The small toroidal 

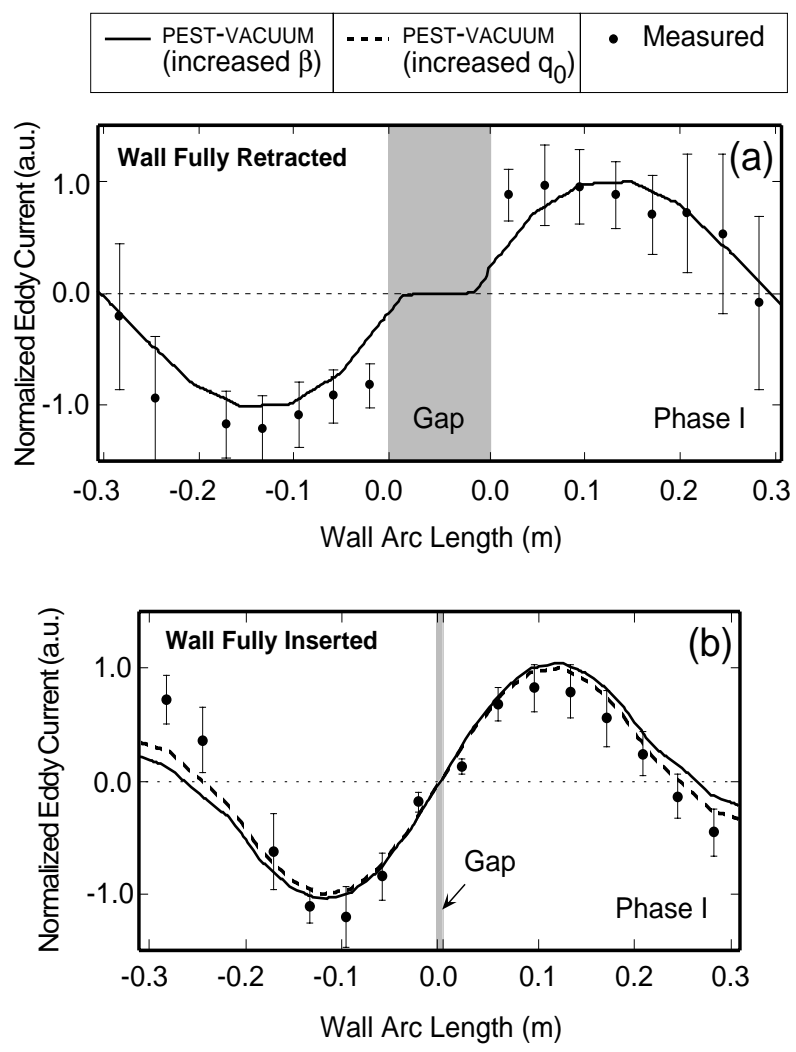

FIG. 13. Comparison of measured and predicted eddy currents, showing the poloidal distribution at phase I of Fig. 10(d) for (a) wall fully retracted, discharge 12088 and (b) wall fully inserted, discharge 11491.

eddy currents measured on the vacuum side of the wall follow paths of lowest inductance that run mainly along the poloidal direction.

In Fig. 15 the detailed comparisons of measurements and predictions are carried out for both wall inserted and wall retracted cases at two phases. At phase II the predicted poloidal distribution on the plasma facing side of one wall segment sums to zero. At phase III $\left(90^{\circ}\right.$ apart $)$, the predicted distribution is mostly unipolar. Again the agreement is generally good, with most significant differences appearing in phase III.

To understand these deviations we first need to note that, in the code calculations, the poloidal distribution of the toroidal eddy current always sums to zero on the plasma facing side of the wall (Fig. 13) and stays close to zero on the vacuum side (Fig. 15). The presence of a gap where the code has a bulge implies that when a mostly unipolar current distribution is predicted to occupy the entire plasma facing side of one wall segment (as in Figs 13, 15(b) and $15(\mathrm{~d})$ ), deviations must appear in the measured pattern to allow for the return currents. From the measurements we see that these return currents tend to flow mostly:

(a) Near the edges of the segment in the wall fully inserted configuration (Fig. 15(d) at wall arc lengths of 0.3 and 0.65 ),

(b) On the vacuum side of the wall in the wall fully retracted configuration (Fig. 15(b) for wall arc lengths greater than 0.3).

In the latter case it is possible that these return currents decrease the (small) stabilizing radial field predicted by the code in correspondence to the outer midplane gap.

\section{SUMMARY AND CONCLUSIONS}

Plasma discharges near the marginal stability boundary to $n=1$ external kink modes have been formed in HBT-EP with the same minor radius but different wall configurations: wall fully retracted and wall fully inserted. The plasma formation technique combines a rapid formation startup with a slow current rampup to create high- $\beta_{\mathrm{N}}$ plasmas with broad current profiles and clear sawtooth oscillations that indicate values close to unity for the safety factor at the magnetic axis.

The equilibria have been carefully reconstructed, using the numerous available external and internal magnetic measurements, an eigenmode representation of the equilibrium eddy currents induced in the segmented wall and vacuum chamber, and the assumption $1.02<q_{0}<1.04$ during sawtooth cycles.

The accuracy of the procedure is supported by the results of several tests. Good agreement has been shown between measured soft $\mathrm{X}$ ray profiles and simulated profiles obtained using the reconstructed pressure distributions. The values of $\beta_{\mathrm{N}}$ and $q_{\text {edge }}$ calculated in the equilibrium reconstructions are in good agreement with the same quantities estimated from toroidal force balance. When instabilities develop, the observations of stability thresholds are consistent with the predictions of ideal MHD theory. The mode structure and the growth rate of the fast growing modes, causing disruptions when the wall is fully retracted, are in good agreement with the mode structure and the growth rate of the predicted ideal external kink mode. The patterns of the instability induced eddy currents measured on the wall for the two different wall positions are in agreement with the patterns calculated for a model wall by the stability codes. 


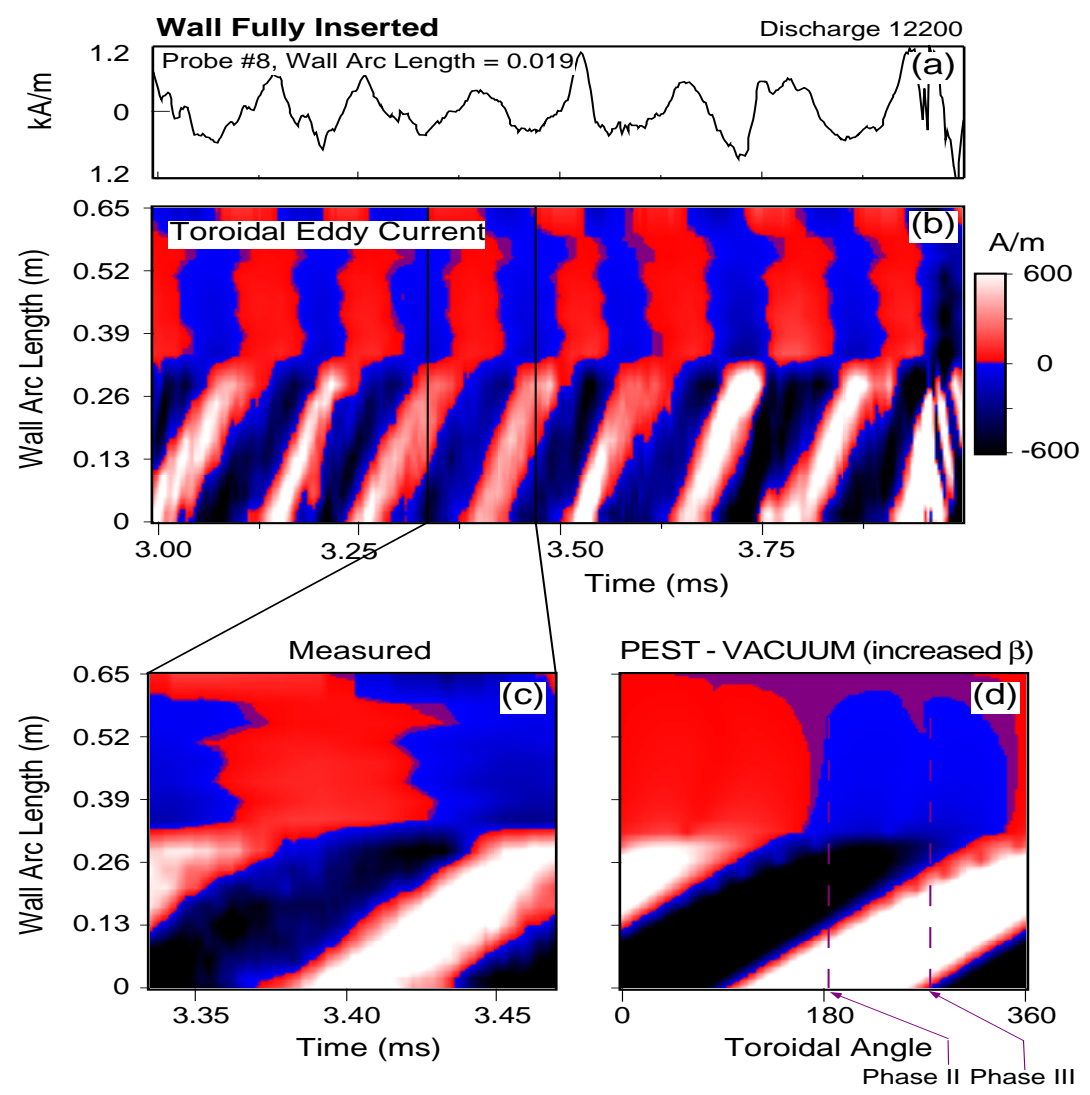

FIG. 14. Toroidal component of the instability induced eddy currents on the plasma facing and vacuum side of one wall segment during discharges with the wall fully inserted: (a, b, c) time evolution; (d) pattern calulated by PEST1VACUUM. Discharge 12200.

This last result leads to two important conclusions. The first is that with respect to high frequency fluctuations the resistive wall behaves as an ideal wall, i.e. the instability induced eddy currents do not leak through the wall. The second is that the segmentation of the wall does not prevent, at least away from the edges, the eddy currents from reproducing the helical patterns predicted for a continuous wall. These eddy currents can account for the stabilizing effect of the close fitting wall.

When the wall is fully inserted, the fast growing ideal mode predicted for no wall by the stability codes, $\gamma_{\text {PEST }}^{-1} \approx 30 \mu \mathrm{s}$, is stabilized. However, a slowly growing instability $\left(\gamma^{-1} \geq 600 \mu \mathrm{s}\right)$ is observed when the wall is fully inserted, which is clearly different in nature from the fast ideal mode observed when the wall is fully retracted. Although the measurements of the eddy current patterns agree with the predictions for an ideal external kink, the reproducible slow growth rate is consistent with a resistive mode. The analysis of the mode structure of this slowly growing instability suggests a combination of a saturated internal tearing mode with a slowly growing external resistive wall mode. These instabilities have a sufficiently long time-scale that their growth could be controlled by active feedback techniques. Initial experiments on active feedback control have been carried out on HBT-EP and the results are described in Ref. [34].

\section{ACKNOWLEDGEMENTS}

The authors gratefully acknowledge the invaluable contributions from J. Manickam, M. Chance, S. Sabbagh and M. Okabayashi. Furthermore, the authors are indebted to the HBT-EP technical staff for continuous support, in particular M. Cea, N. Rivera and E. Rodas. 


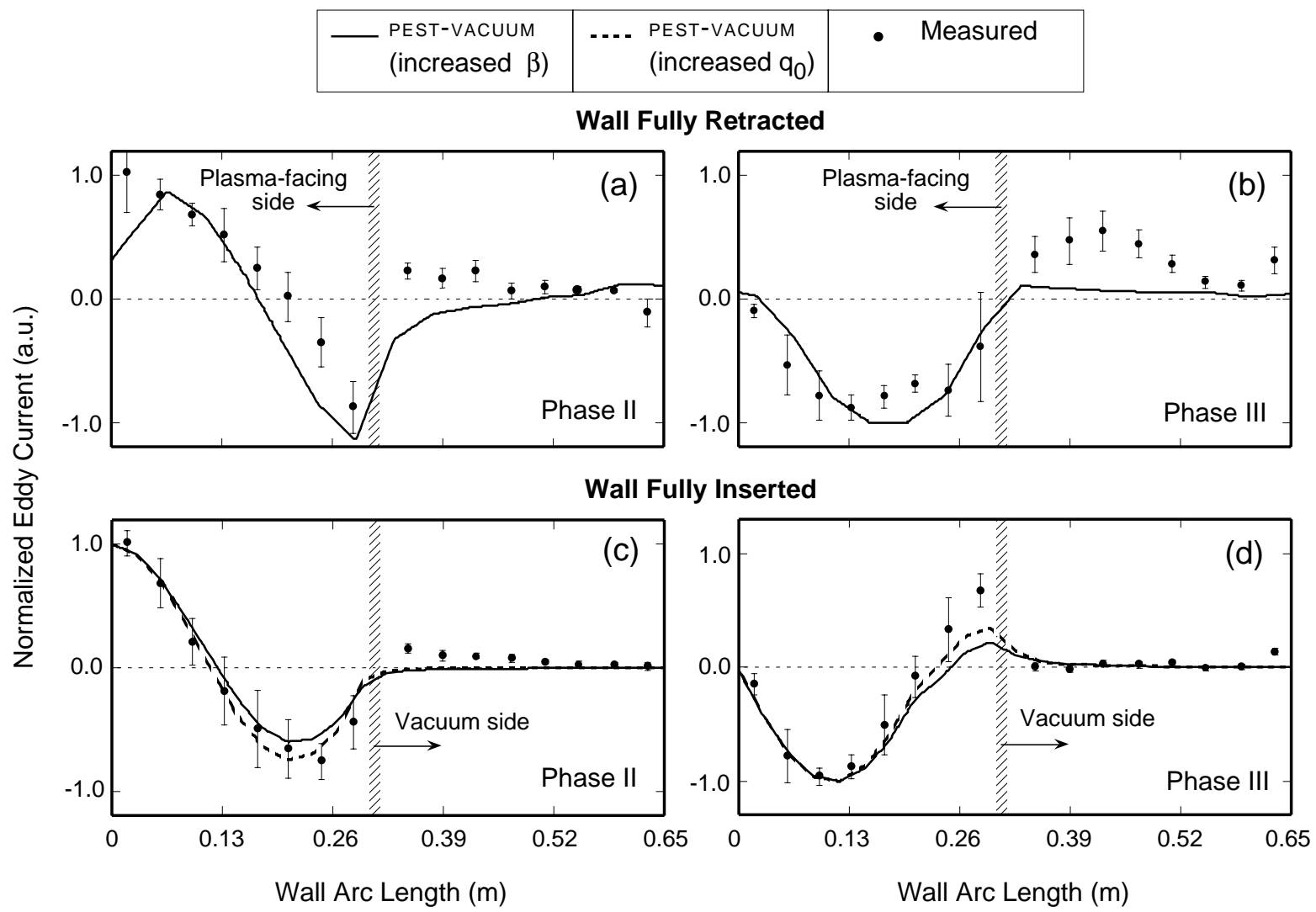

FIG. 15. Comparison of measured and predicted eddy currents, showing the poloidal distributions at phases II and III of Fig. 14(d): (a) and (b) for the wall fully retracted discharge 12200; (c) and (d) for the wall fully inserted discharge 12094 .

\section{REFERENCES}

[1] TROYON, F., et al., Plasma Phys. Control. Fusion 26 (1984) 209.

[2] SHAFRANOV, V.D., Sov. Phys. - Tech. Phys. 15 (1970) 175.

[3] KESSEL, C., et al., Phys. Rev. Lett. 72 (1994) 1212.

[4] MENARD, J.E., et al., Nucl. Fusion 37 (1997) 595.

[5] NAJMABADI, F., CONN, R.W., in Plasma Physics and Controlled Nuclear Fusion Research 1992 (Proc. 14th Int. Conf. Würzburg, 1992), Vol. 3, IAEA, Vienna (1993) 295.

[6] TURNBULL, A.D., et al., Phys. Rev. Lett. 74 (1995) 718.

[7] ROSEnBluth, M.N., et al., in Plasma Physics and Controlled Nuclear Fusion Research 1994 (Proc. 15th Int. Conf. Seville, 1994), Vol. 2, IAEA, Vienna (1995) 517.

[8] FREIDBERG, J.P., Ideal Magnetohydrodynamics, Plenum Press, New York and London (1987).

[9] NALESSO, G.F., COSTA, S., Nucl. Fusion 20 (1980) 443.

[10] GIMBLETT, C.G., Nucl. Fusion 26 (1986) 617.

[11] HENDER, T.C., et al., Nucl. Fusion 29 (1989) 1279.
[12] BETTI, R., FREIDBERG, J.P., Phys. Rev. Lett. 74 (1995) 2949

[13] FINN, J.M., Phys. Plasmas 2 (1995) 3782.

[14] BOOZER, A.H., Phys. Plasmas 2 (1995) 4521.

[15] FITZPATRICK, R., Phys. Plasmas 1 (1994) 2931.

[16] BONDESON, A., WARD, D.J., Phys. Rev. Lett. 72 (1994) 2709.

[17] STRAIT, E.J., et al., Phys. Rev. Lett. 74 (1995) 2483.

[18] OKABAYASHI, M., et al., in Plasma Physics and Controlled Nuclear Fusion Research 1986 (Proc. 11th Int. Conf. Kyoto, 1986), Vol. 1, IAEA, Vienna (1987) 275.

[19] VIJAYA SANKAR, M.K., et al., J. Fusion Energy 123 (1993).

[20] IVERS, T.H., et al., Phys. Plasmas 3 (1996) 1926.

[21] LAO, L.L., et al., Nucl. Fusion 25 (1985) 1611.

[22] SABBAGH, S.A., et al., in Fusion Energy 1996 (Proc. 16th Int. Conf. Montreal, 1996), Vol. 1, IAEA, Vienna (1997) 921.

[23] MANICKAM, J., Princeton Plasma Physics Lab., NJ, personal communication, 1997.

[24] TURNBULL, A.D., On the Operational Ideal $\beta$ Limit in Sawtoothing Discharges, Rep. GA-A22807, 
Natl Tech. Information Service, Springfield, VA (1998).

[25] LAZARUS, E.A., et al., Nucl. Fusion 30 (1990) 111.

[26] NAGAYAMA, Y., et al., Nucl. Fusion 24 (1984) 1243.

[27] GRIMM, R.C., et al., in Methods in Computational Physics, Vol. 16 (KILLEEN, J., Ed.), Academic Press, New York (1976) 253.

[28] MANICKAM, J., et al., Comput. Phys. Commun. 24 (1981) 355.

[29] CHANCE, M.S., Phys. Plasmas 4 (1997) 2161.

[30] IVERS, T.H., et al., in Plasma Physics and Controlled Nuclear Fusion Research 1990 (Proc. 13th Int. Conf. Washington, DC, 1990), Vol. 1, IAEA, Vienna (1991) 573.

[31] HUTCHINSON, I.H., in Principles of Plasma Diagnostics, Cambridge University Press, Cambridge (1987) 26.

[32] OKABAYASHI, M., et al., Nucl. Fusion 36 (1996) 1167.
[33] TODD, A.M.M., et al., Phys. Rev. Lett. 38 (1977) 826.

[34] NAVRATIL, G.A., et al., Phys. Plasmas 5 (1998) 1855.

(Manuscript received 12 September 1997

Final manuscript accepted 24 March 1998)

E-mail address of A.M. Garofalo:

garofalo@gav.gat.com

Note added in proof

Present address of A.M. Garofalo:

General Atomics,

San Diego, California, USA

Subject classification: C0, Te; D3, Te; E0, Te 